\title{
HACIA UNA CARACTERIZACIÓN DE LOS ESTILOS DE PLANEACIÓN DIDÁCTICA: UNA APROXIMACIÓN COGNITIVA ${ }^{1}$
}

\author{
TOWARDS A CHARACTERIZATION \\ OF TEACHER PLANNING STYLES: \\ A COGNITIVE APPROXIMATION
}

\author{
Diego Degetau Arsuaga ${ }^{2}$ \\ https://orcid.org/0000-0001-6884-5710 \\ Luis Medina Gual ${ }^{3}$ \\ https: / / orcid.org/0000-0002-6783-606X
}

Recibido: febrero 3, 2020 - Aceptado: mayo 7, 2020.

\section{RESUMEN}

Han pasado más de 70 años desde que Ralph Tyler desarrolló su teoría sobre la planeación didáctica. Desde entonces, son muchos los autores que han propuesto modelos prescriptivos que guíen la planeación de la enseñanza, reconociendo por qué la planeación didáctica está en el centro de cualquier docente efectivo. El presente estudio tiene como objetivo caracterizar los estilos de planeación didáctica de docentes de educación básica y media superior de una red de colegios particulares. Así, desde una aproximación cognitiva a la estilística de la planeación didáctica, que consiste en el estudio de la toma de decisiones realizadas por el docente en torno a los elementos didácticos que constituyen un plan didáctico, se confirma la fuerte relevancia de la propuesta de Tyler, 70 años después de su publicación original. Se identificó que el $60.62 \%$ de los docentes estudiados son altamente congruentes con dicha racionalidad, mientras que los resultados de los docentes restantes, varían su estilo de planeación para alinearse a una planeación basada en estándares o en competencias. Así mismo, se encontró tras un análisis de componentes principales, una varianza explicada del 74\%. Esto brinda evidencias sobre la validez de la aproximación metodológica.

Palabras clave: perfil del profesor, práctica docente, planeación didáctica.

1 Se agradece el apoyo de la Red de Colegios Semper Altius y la Oficina Central de la Red de Colegios Semper Altius para la realización del estudio.

2 Licenciado en Pedagogía, Panamericana. Co-fundador \&amp; Director General de Lumbria Consultores. ddegetau@gmail.com

3 Doctorado en Evaluación Educativa, Universidad Anáhuac, México. Maestría en Educación y Desarrollo, Universidad Iberoamericana. Sistema Nacional de Investigadores. Departamento de Educación, Universidad Iberoamericana CDMX. luis.gual@ibero.mx medinagual@gmail.com 


\section{ABSTRACT}

It's been 70 years since Ralph Tyler developed his theory of curriculum design. Since then, there have been many authors who have proposed prescriptive models that guide teacher planning, recognizing why teacher planning is at the center of any effective teacher. This study seeks to characterize the teacher planning styles of teachers from a network of private schools. From a cognitive approximation of teacher planning styles, which consists of studying teacher's decision-making regarding the elements that constitute their lesson plan, it was confirmed the strong relevance that Tyler's proposal has after 70 years from its original publication. About $60.62 \%$ of the studied teachers were identified as highly congruent with such rationality. In contrast, the other studied teachers vary their planning style aligning themselves with a standard or competencies-based planning. Likewise, after a principal component analysis, an explained variance of $74 \%$ was found, bringing evidence about the validity of methodological approximation.

Key words: Lesson Plans, Academic Planning, Curriculum Planning.

\section{INTRODUCCIÓN}

La planeación didáctica, a lo largo de la literatura especializada, es llamada de diversas formas, sin especificar la variación entre sus connotaciones. El término "planeación didáctica» alude a dos sentidos: planeación como proceso y planeación como producto (Monroy, 2009). El primero refiere a todas las decisiones que el docente debe tomar para llegar al segundo, el documento que instruye su actuar. En este sentido, la planeación es un proceso donde intervienen diversos elementos que organizan el proceso de enseñanza-aprendizaje. La labor del docente es ponderar dichos elementos al contrastarlos con sus propias competencias docentes, las del alumnado, los contenidos por enseñar y la interacción entre ellos para que, de esta manera, se alcancen los objetivos educativos generales propuestos. Un docente tiene, como beneficios de la planeación, el pensar deliberadamente sobre lo que se pretende que ocurra en el aula y que aprendan los estudiantes, evaluar el conocimiento que el docente mismo posee sobre el contenido o los aprendizajes por enseñar $y$, finalmente, brinda confianza para ejecutar la planeación (Ashcraft, 2014).

Durante el proceso de planeación, los docentes emplean diferentes «elementos didácticos» con la finalidad de articular las diversas decisiones y momentos que el docente ejecutará en el aula. Para Monroy (2009), estos elementos son:

- Cuáles son las características del grupo al que va dirigida la enseñanza: diagnóstico del grupo de aprendizaje.

- Qué se define como imprescindible que aprenda el alumnado al finalizar el programa: objetivos educativos.

- Cuáles son los conocimientos que el alumnado debe aprender para cumplir con los objetivos: contenidos de aprendizaje.

- Cómo se deben trabajar los contenidos en el contexto áulico: métodos, estrategias y actividades didácticas.

- De cuánto tiempo se dispone, y cómo se empleará ese tiempo para cada contenido y estrategia didáctica: tiempo didáctico. 
- Qué medios materiales o inmateriales se necesitan para realizar las actividades planeadas: recursos didácticos.

- Cómo se sabrá si se alcanzaron los objetivos educativos: evaluación del aprendizaje.

Autores como Díaz-Barriga Arceo (2004) y Zabalza (2004) argumentan que se pueden agregar sub-elementos didácticos a partir de los elementos generales antes mencionados. Entre ellos, las preguntas que se suelen plantear entre los docentes son:

- Cómo se relacionan los conocimientos que el alumnado debe aprender con la realidad social que vive: relación del contenido con la vida real.

- Qué debe hacer el estudiante para encuadrar sus aprendizajes materialmente: proyecto de la clase.

Estas son las decisiones que el docente debe tomar a priori a su clase. Es decir, su planeación debe contemplar estos elementos para que se concrete en una planeación didáctica. Para los fines de la presente investigación, estos serán los elementos estudiados a lo largo del documento.

\section{LA APROXIMACIÓN COGNITIVA AL ESTUDIO DE LA PLANEACIÓN DIDÁCTICA}

La inspiración detrás de la aproximación cognitiva parte del estudio de los modelos prescriptivos de planeación de la enseñanza. Desde que Ralph Tyler publicó su famosa racionalidad (1950), se han propuesto una amplia variedad de modelos sobre cómo los docentes deberían planear sus clases. Cada modelo propone una forma distinta en que los elementos didácticos (unos omiten algunos, otros agregan más elementos) deben ser pensados y secuenciados en la toma de decisiones.

En este sentido, la aproximación cognitiva estudia todos los procesos que suceden al interior de la mente del docente. Esta forma de estudio se consolida a partir de las investigaciones sobre el pensamiento docente (Clark y Yinger, 1979), la propuesta de Ives Chevallard sobre la transposición didáctica (1989) y los modelos existentes de diseño curricular, propuestos en el último siglo. Coherente con lo anterior, la aproximación cognitiva se enfoca en la toma de decisiones alrededor de los elementos didácticos que constituyen un plan. Para la presente investigación, el interés detrás de la aproximación cognitiva es comprender cómo es que los docentes ordenan y priorizan cada uno de los elementos didácticos antes mencionados al realizar una planeación. Precisamente en esta tónica, el ejercicio de contextualizar el saber en la realidad educativa propone de manera directa comprender el rol del resto de los elementos didácticos en la definición del plan (Ramírez, 2005).

\section{LOS MODELOS DE PLANEACIÓN DIDÁCTICA}

Mientras que en la primera mitad del siglo XX, ingenieros y administradores de plantas industriales desarrollaban los principios básicos de la administración científica, Franklin Bobbit (1918) intentaba extender dichos principios al campo de la educación (Koeller y Thompson, 1980). Inspirado en las ideas de Bobbit, Ralph Tyler (1950) desarrolla su teoría 
sobre la planeación educativa que consiste en separar los fines y los medios educativos. Esta separación prioriza los objetivos sobre cualquier otra actividad debido a que, al hacerlo, los objetivos «se vuelven el criterio por el cual los materiales se seleccionan, los contenidos se describen, los procedimientos instruccionales se desarrollan, y los exámenes se preparan» (p.3).

Así es como, posteriormente, Benjamin Bloom (1956) llega al cometido de clarificar el modelo de Tyler fundamentando la necesidad de crear taxonomías que permitan clasificar cualquier tipo de aprendizaje para que pudieran plasmarse en la redacción de cualquier objetivo educativo. A partir de este trabajo surgen -y siguen surgiendo- una serie de taxonomías de todo tipo de aprendizaje. Por mencionar algunas,

en el plano del dominio cognoscitivo las de John R. Anderson, A.J. Romiszowsky y Roberto J. Marzano; del dominio psicomotor como las de Seymour, Guilford y Gagné; del dominio afectivo como las de W. French, Marting y Briggs y García Hoz; matemáticas de Freeman y Crow; ciencias como Klopfer; laborales de Bladwin; sobre tecnologías como la de Tornel; etcétera (Medina Gual, 2011, p. 55).

El modelo prescriptivo de Tyler - hoy conocido como racionalidad tyleriana- mantiene su popularidad como modelo primario en la enseñanza de la planeación didáctica entre varias escuelas de educación (cfr. Koeller y Thompson, 1980; Kyung Ko, 2012). John (2006) argumenta que su permanencia tras tantas décadas radica en su «elegante simplicidad» (p. 486) que trasciende los climas políticos de cualquier época. Comenta que la lógica detrás el apoyo de la racionalidad tyleriana parte de preguntarse:

\begin{abstract}
¿Cómo podemos saber si estamos alcanzando nuestras metas si no las hemos especificado claramente en un primer momento? ¿Cómo es posible analizar el proceso de enseñanza-aprendizaje si no lo dividimos en todas las partes que lo componen? ¿Cómo podemos diseñar, planear o implementar cualquier cosa si no pasamos por el proceso racional de formular objetivos, decidir estrategias, seleccionar recursos, organizar actividades, diseñar su implementación y evaluar sus resultados? (John, 2006, p. 486).
\end{abstract}

Su concepción estructuralista, señala Cherryholmes (1988, citado en John, 2006, p. 486), se basa en «una forma sistemática de pensamiento sobre procesos e instituciones donde cada parte de un sistema define y es definido por otras partes».

Por otro lado, Hilda Taba (1962) propone un modelo prescriptivo de planeación donde amplía la racionalidad tyleriana y agrega un elemento: el diagnóstico de necesidades. En su modelo argumenta que el diagnóstico de necesidades toma lugar antes de que se formulen los objetivos y que ello contribuye a inspirar, en mejor medida, la formulación de los objetivos educativos y la definición de las estrategias de enseñanza. Al realizar un diagnóstico previo a la definición de cualquier otro elemento, Taba explica que de esta manera es posible para los docentes tomar decisiones más pertinentes al planear.

En cambio, autores como Macdonald (1965) y Eisner (1967) ponen en duda a la racionalidad tyleriana y se preguntan si en realidad la primera decisión de un docente es definir los objetivos y después proceder a decidir sobre las actividades, los recursos, la evaluación y cualquier otro elemento (Zahorik, 1975). Sugieren que existen ciertos propósitos educativos que no pueden ser descritos conductualmente y que, al tener que definirlos de tal manera, podría imponerse una formalidad demasiado rígida a expensas de la creatividad en la enseñanza del docente (Koeller y Thompson, 1980). Así, proponen 
un modelo de planeación donde la primera decisión que realiza el docente debe ser el tipo de actividades que deberían hacer para promover el aprendizaje en su salón de clases. Este modelo sugiere que los fines se integren con los medios, ya que al identificar primero el tipo de actividades con que se debería de proveer la experiencia educativa, los objetivos terminan surgiendo de la actividad misma (Macdonald, Wolfson y Zaret, 1973). Zahorik (1975, p. 134) comenta: «[...] aunque la prescripción de Macdonald sea menos conocida que la de Tyler y posiblemente tenga menos seguidores, su modelo bien podría describir lo que de hecho hacen los docentes».

No fue sino hasta 25 años después, que surgió un cuarto y último modelo prescriptivo en la planeación de la enseñanza. Tras el acta No child left begind, del gobierno estadounidense (2001), se fue propugnando un movimiento de igualdad de oportunidades educativas que hoy conocemos como el movimiento de estándares educativos. En última instancia, el movimiento respondía a las necesidades de equidad educativa de la época. Los estándares consisten en establecer el mínimo indispensable que todo estudiante debería ser capaz de conocer, hacer o ser en un momento determinado, con el fin de asegurar la formación de la persona (Medina Gual, 2011). En esta tónica, los estándares implican «una selección muy rigurosa, y socialmente validad, del conjunto de conocimientos al que todos los alumnos tendrán el derecho irrenunciable de acceder, independientemente de su ámbito sociocultural de pertenencia» (Ferrer, 2006, citado en Medina Gual, 2011, p. 58).

Así pues, surge el modelo denominado Understanding by Design o Backward design (en español, «comprendiendo el currículo» o "planeación al revés» o "planeación hacia atrás») de Grant Wiggins y Jay McTighe (2005). La lógica seguida por este modelo podría considerarse «al revés» de lo que propone Tyler. La planeación comienza definiendo los resultados de aprendizaje esperados de los alumnos y los mecanismos de evaluación que permitirán al docente identificar el logro de esos aprendizajes. Por lo tanto, como contraste a los modelos anteriores, este modelo antepone la evaluación previamente a considerar las estrategias de enseñanza. De esta manera, el modelo obliga a que los docentes piensen primero en el aprendizaje deseado, promoviendo una mayor intencionalidad en la planeación didáctica y fomentando la premisa central detrás de la equidad educativa: si se desea que el alumno ejecute una tarea determinada, el docente debe pensar en qué hacer para que todos los estudiantes logren elaborarla con eficacia (Medina Gual, 2011).

Jack C. Richards (2013) sintetiza los modelos mencionados en su propia tipología de la planeación: forward design, central design y backward design (en español, "planeación hacia delante», "planeación central»y «planeación al revés»). Para comprender su caracterización, limita la planeación a la decisión sobre tres elementos: el contenido, que equivale al temario; el proceso, que corresponde a la metodología de enseñanza; y los resultados de aprendizaje, que equivalen a los mecanismos de evaluación. Por lo tanto, distingue los modelos a partir de la lógica que sigue la relación entre variables. El forward design significa planear pasando del contenido al proceso y de ahí al resultado; similar a la racionalidad tyleriana clásica. El central design representa planear empezando con el proceso y que de él se derive el contenido y los resultados; análogo a la propuesta de Macdonald (1965). Por último, el backward design señala empezar con el resultado y después lidiar con el proceso y el contenido; equivalente al modelo de McTighe y Wiggins (2005). Estos se pueden representar de la siguiente manera: 
Figura 1. El proceso de planeación central

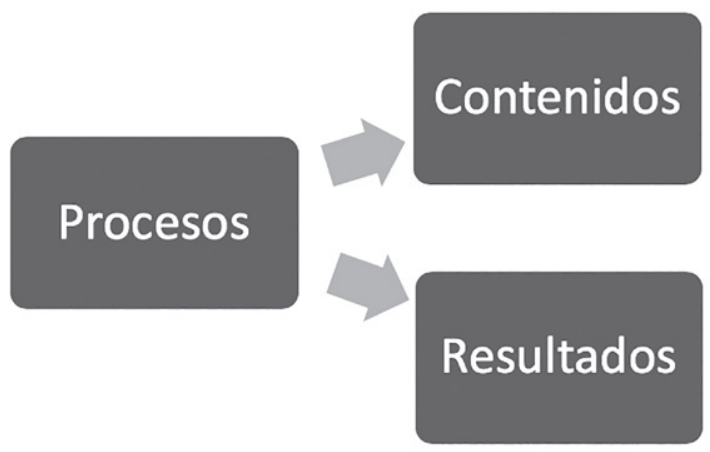

Figura 2. El proceso de planeación backards

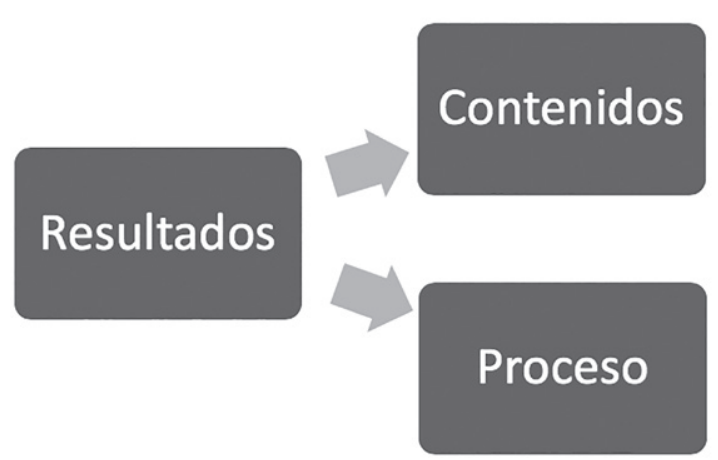

A partir del método de aproximación cognitiva propuesto, el presente artículo busca caracterizar los estilos de planeación didáctica de docentes de educación básica y media superior de una red de colegios particulares. Para ello, se analiza el orden en el que se priorizan los procesos cognitivos de planeación didáctica de los docentes, buscando identificar los estilos de planeación, describirlos, y comparar los hallazgos obtenidos con los modelos teóricos de planeación didáctica planteados anteriormente.

\section{METODOLOGÍA}

Este es un estudio de corte cuantitativo de tipo ex post facto, de alcance exploratorio, descriptivo y correlacional. Las variables e instrumento empleado se pueden consultar en los documentos anexos y parten de los elementos y subelementos de la planeación mencionados en la introducción. La población meta consta de docentes pertenecientes a una red de colegios particulares confesionales, con presencia en México y un colegio en El Salvador. A continuación, se describe el proceso de elaboración y validación del instrumento empleado para el presente estudio.

\section{Proceso de validación del instrumento}

El instrumento diseñado parte de la literatura revisada y un proceso de entrevistas no estructuradas a docentes y especialistas en investigación sobre prácticas de planeación, así 
como análisis de contenido de la planeación de docentes. El proceso de validación del instrumento se realizó en tres etapas. Una inicial donde se elaboraron $\mathrm{N}=10$ entrevistas cognitivas de un instrumento preliminar a docentes de un colegio de la Ciudad de México, perteneciente a la red de colegios evaluada $(\mathrm{n}=1$ docente de preescolar, $\mathrm{n}=2$ docentes de primaria, $\mathrm{n}=3$ docenes de secundaria y $\mathrm{n}=4$ docentes de media superior). En la segunda etapa, a partir de la documentación de la retroalimentación de las entrevistas cognitivas, se acudió a dos investigadores educativos de distintas instituciones de educación superior que fungieron como jueces otorgando comentarios puntuales sobre el instrumento. Con base a ello, se conformó una nueva versión del instrumento a aplicarse.

El tercer procedimiento de validación consistió en enviar el instrumento a un colegio no perteneciente a la red de colegios, ubicado en Xalapa, Veracruz. N=35 docentes contestaron el instrumento en esta aplicación. El propósito de esta validación fue comprobar la comprensión de los docentes en la aplicación auto-gestionada del instrumento al realizar los análisis de psicométricos de confiabilidad clásica y validez correspondientes. El instrumento final puede consultarse en los anexos.

\section{RESULTADOS}

\section{Descripción de la muestra}

La muestra final estuvo compuesta por $\mathrm{n}=2120$ docentes de educación básica y media superior, pertenecientes a n=66 colegios de la Red de Colegios Semper Altius. Los colegios están ubicados en casi todos los estados de la República Mexicana, y uno se encuentra en El Salvador. Entre la información relevante a tomarse en consideración son los niveles educativos en que los docentes que contestaron imparten clases, siendo $22.45 \%$ en preescolar, $33.49 \%$ en primaria (K1-K6), 21.42\% en secundaria (K7-K9) y $18.35 \%$ en bachillerato (K10-K12).

En cuanto al sexo de los respondientes, la participación de mujeres es del $80.61 \%$ de la muestra, mientras que la de los hombres es del 19.38\%. Las siguientes tablas indican la experiencia en enseñanza (media=14.42, sd=9.5) y el máximo grado de estudios de los docentes.

\section{Tabla 1. Distribución de los años de experiencia de enseñanza de la muestra $(n=2120)$}

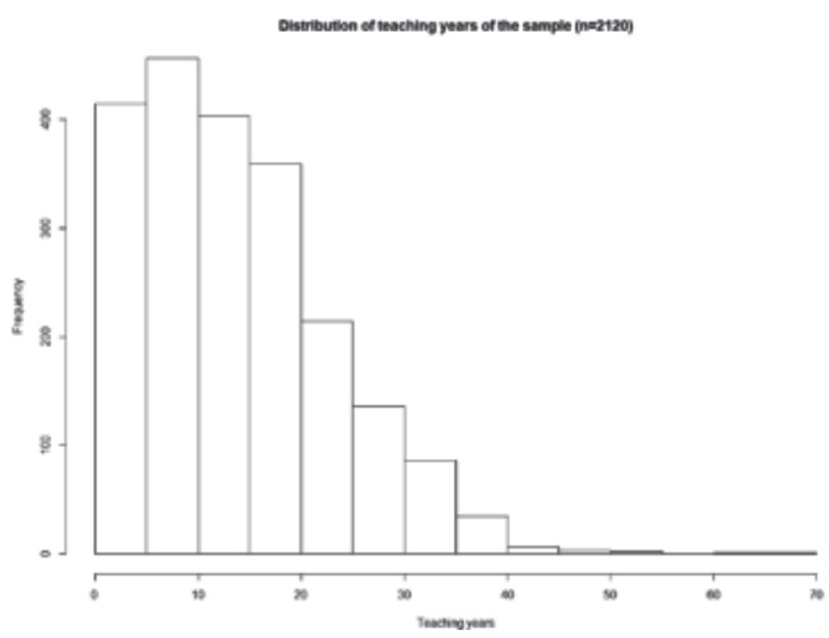


Tabla 2. Distribución del máximo grado de estudios de la muestra $(n=2120)$

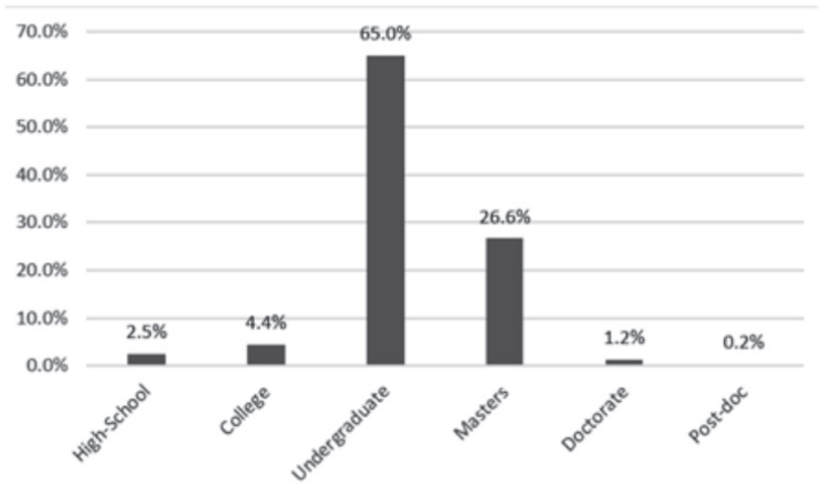

Finalmente, la siguiente tabla muestra la distribución de la muestra basada en el área disciplinar de enseñanza de los docentes (un docente puede enseñar en más de un área). Cada docente seleccionaba las áreas disciplinales en las que se consideraba experto.

\section{Tabla 3. Distribución del área disciplinar} de enseñanza de la muestra $(n=2120)$

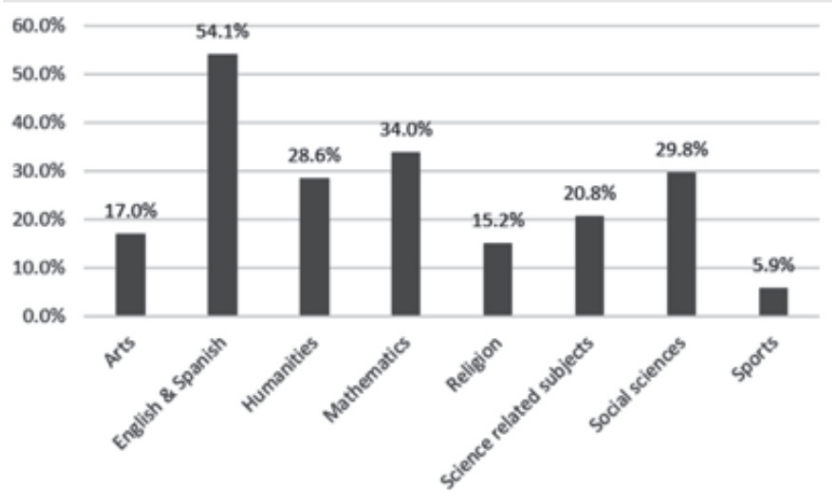

\section{Análisis inferenciales}

Para mostrar los diferentes estilos de planeación didáctica que se hallaron, la siguiente tabla señala la distribución de los procesos didácticos, según el orden de ejecución pensado por los docentes: 
Tabla 4. Procesos de planeación ordenados según el tiempo o momento de su ejecución ( $n=2120)$

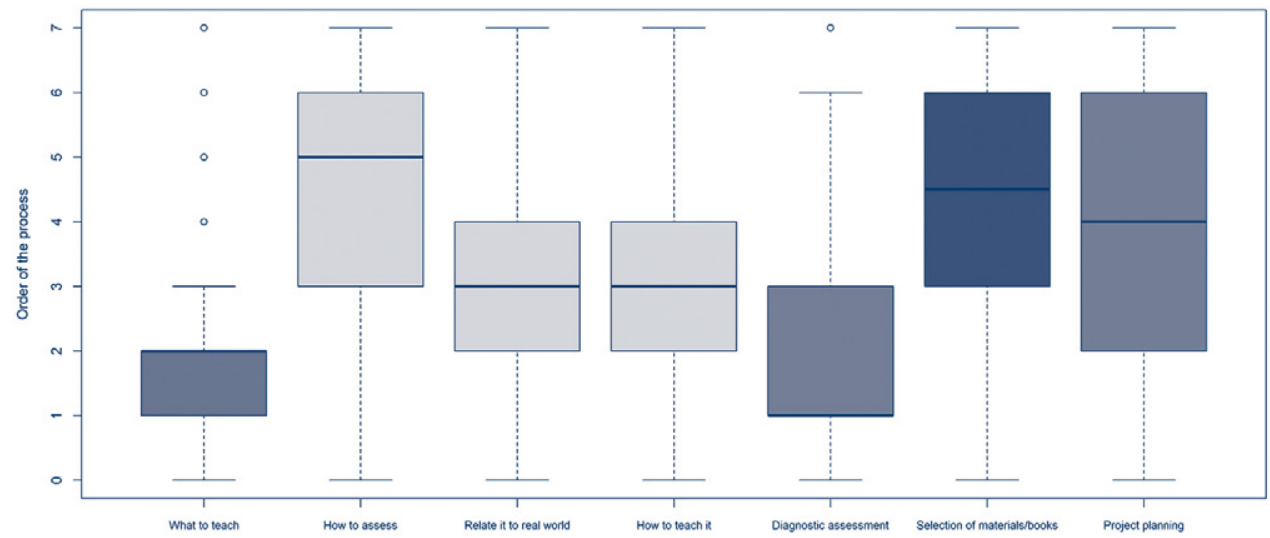

Como muestra la tabla anterior, los dos procesos de planeación que parecen ser lo primero que realizan los docentes al planear son: la definición de «qué enseñar» (la selección de los objetivos de aprendizaje o sus resultados) y la realización de una «evaluación diagnóstica» de los estudiantes. En un segundo momento, los docentes consideran la definición de «cómo enseñar» y el llevar cabo la «relación del contenido con contextos de la vida real».

Por último, tres procesos de planeación parecen realizarse simultáneamente: la definición y planeación del «proyecto», la «selección de materiales y libros», y la definición de "cómo evaluar». Es importante notar que mientras contestaban el cuestionario, los docentes podrían haber seleccionado la opción «No lo hago», lo cual significa que el docente no considera el proceso en sus planes, o haber indicado que realiza uno o más procesos al mismo tiempo. En un primer intento de analizar la relación entre los diferentes procesos de planeación, se realizó un análisis de correlaciones. Debido a la naturaleza ordinal de las variables, se computó una correlación Spearman-Brown. La siguiente tabla muestra las diferentes correlaciones encontradas con las correlaciones no significantes ( $p>0.01)$ marcadas con una cruz.

Tabla 5. Matriz de correlación de los procesos de planeación (n=2120)

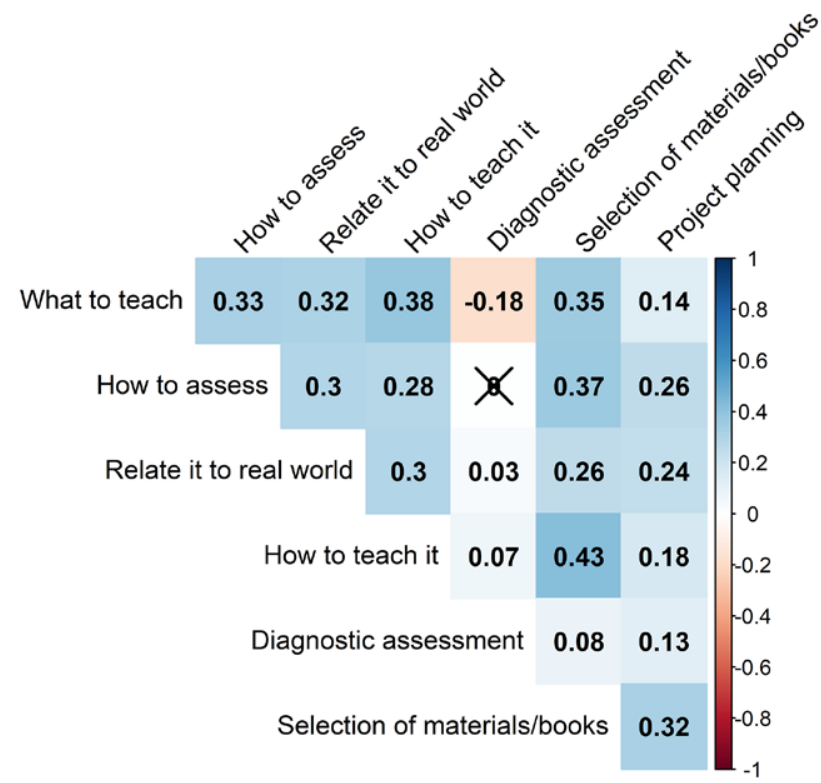


Como se muestra en la tabla anterior, los procesos que sostienen la mayor correlación aparecen con la definición de «qué enseñar». Este proceso parece tener índices medios de correlaciones con todos los procesos excepto con «evaluación diagnóstica». Debido al hecho que la mayoría de los docentes seleccionan «qué enseñar» como su primer o segundo proceso, es lógico inferir que este sostendría altas correlaciones con otros procesos.

Otras correlaciones relevantes son la definición de «cómo enseñar», la «relación del contenido con contextos de la vida real» y la «selección de materiales y libros». Finalmente, existen correlaciones medias entre la definición de «cómo enseñar» y la «selección de materiales y libros», y entre la "planeación de proyectos» y la «selección de materiales y libros». Es relevante notar que la falta de correlación con «evaluación diagnóstica» podría relacionarse con el hecho que la mayoría de los docentes declararon que era lo primero que hacían o que no lo hacían en ningún momento.

Para entender más profundamente los posibles estilos de planeación, se llevó a cabo un análisis de componentes principales (PCA, por sus siglas en inglés). En un primer intento, se usaron los siete procesos de planeación definidos, y se calcularon tanto soluciones ortogonales o de componentes no correlacionados (varimax) como rotaciones no-ortogonales o de componentes que pueden estar correlacionados (oblimin.). De igual manera se calcularon auto valores con la finalidad de indagar cuántos componentes debían de ser extraídos (Eigenvalues). La siguiente tabla representa los Valores Propios:

Tabla 6. Valores propios (de la matriz de covarianza)

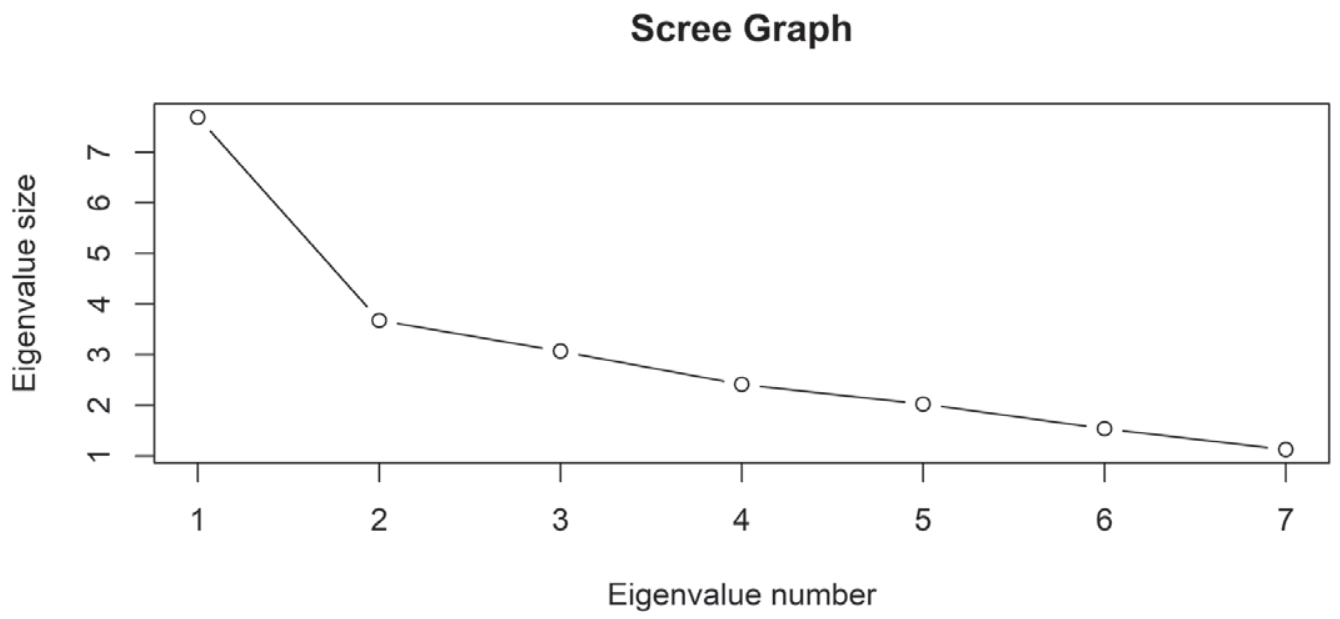

Como se muestra en la tabla anterior, incluso una solución de seis componentes tendría auto valores arriba de 1.0. Sin embargo, para el presente estudio se elegirá una solución de tres componentes, considerando que la revisión de la literatura plantea tres modelos de planeación didáctica (objetivos, competencias y estándares): la racionalidad tyleriana o forward design, la planeación de Macdonald o central design, y la planeación de Wiggins y Mctighe o backward design al elegir tres componentes. Con esto, el análisis de componentes principales indica una varianza explicada del $64 \%$. Las siguientes dos figuras representan las dos soluciones rotadas (varimax vs. oblimin): 


\section{Figura 3. Análisis de componentes principales} con una solución rotada (varimax)

\section{Components Analysis}

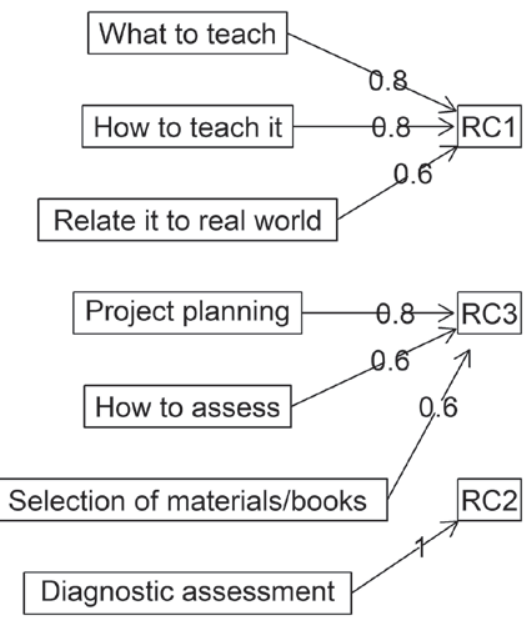

\section{Figura 4. Análisis de componentes principales} con una solución rotada (oblimin.)

\section{Components Analysis}

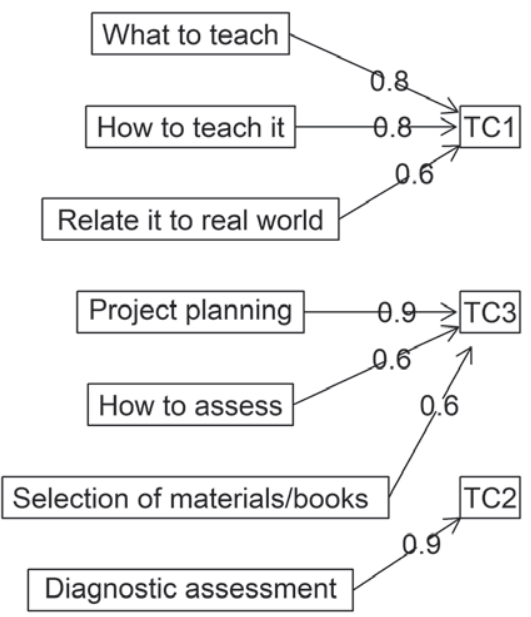

Las dos figuras anteriores permiten discutir la pertinencia del proceso «evaluación diagnóstica» en el análisis. La segunda figura también muestra la pertinencia en considerar una solución no-ortogonal, por la que los factores de los procesos didácticos deberían correlacionarse. En este sentido, las siguientes dos figuras señalan el mismo análisis tras no incluir la «evaluación diagnóstica». 
Figura 5. Análisis de componentes principales con una solución rotada (varimax) y la «evaluación diagnóstica» eliminada

\section{Components Analysis}

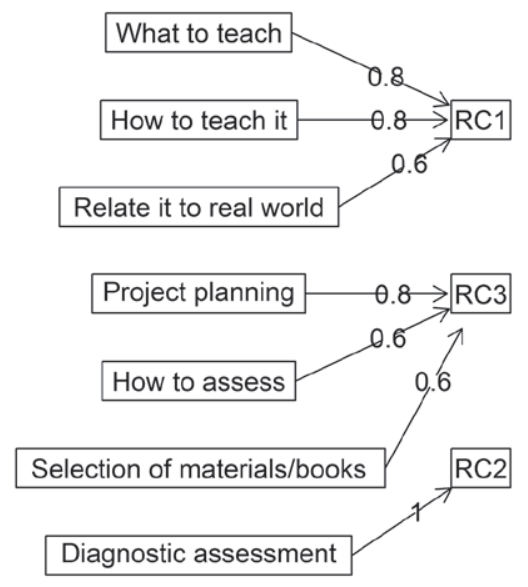

Figura 6. Análisis de componentes principales con una solución rotada (oblimin.) y la «evaluación diagnóstica» rotada

\section{Components Analysis}

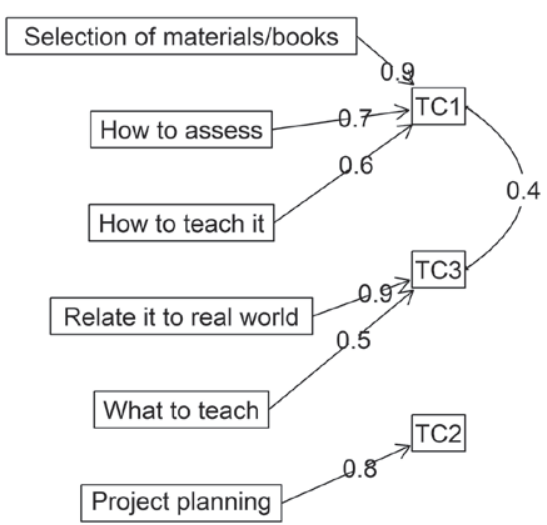

De esta manera, tanto la solución ortogonal como la no-ortogonal muestran la misma estructura en los procesos didácticos, aunque las estructuras de los procesos didácticos no son como según se espera de la teoría. La consistencia de ambas figuras proporciona información esencial sobre posibles estilos de planeación didáctica. Es un hecho sorprendente que el segundo factor detectado no correlaciona con los otros dos. También es importante notar que no correlaciona con el tercer factor.

La solución de la última figura es la que se tendrá en cuenta para los análisis a continuación. En este análisis, el primer factor cuenta por el $27 \%$ de la varianza, el segundo y tercer factor cuentan por el $23 \%$ de la varianza cada uno. En total, el análisis de componentes principales cuenta por una varianza explicada del $74 \%$. 
Por ultimo un análisis de k-medias fue realizado para identificar los grupos de docentes a partir de los diferentes procesos didácticos. Según la recomendación de Everitt y Hothorn (2010, p. 251), una gráfica de la suma de cuadrados dentro de los clusters, por número de conglomerados extraídos, puede ayudar a determinar el número de cluster. Esto se muestra en la siguiente tabla:

\section{Tabla 7. Número de clusters vs. suma de cuadrados dentro del cluster}

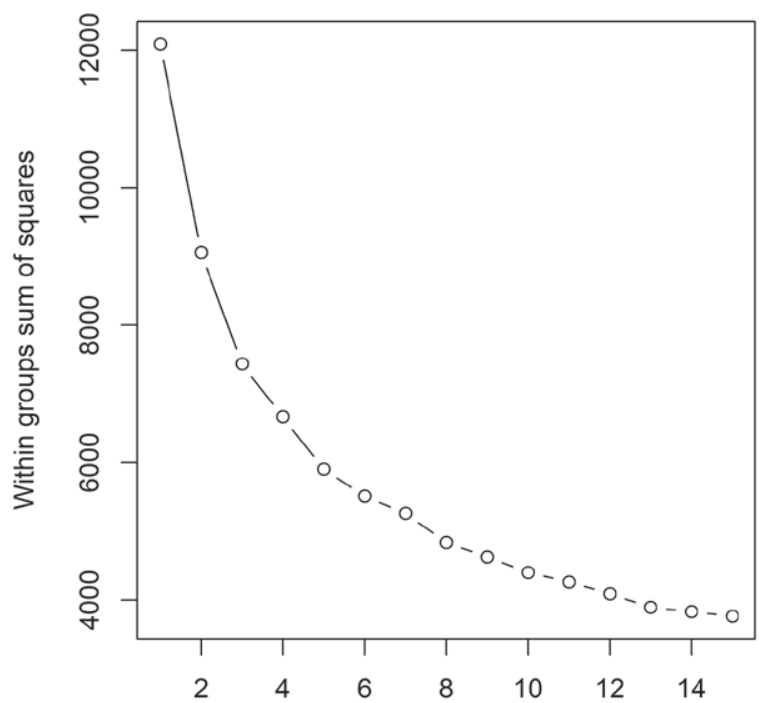

Como se indica en la tabla anterior, el número óptimo de grupos podría ser tres. Basado en esto, se realizó un análisis de clusterización de k-medias. A partir de él, las cuatro siguientes tablas describen los cluster creados:

Tabla 8. Grupo centroide contra la primera y segunda función discriminante

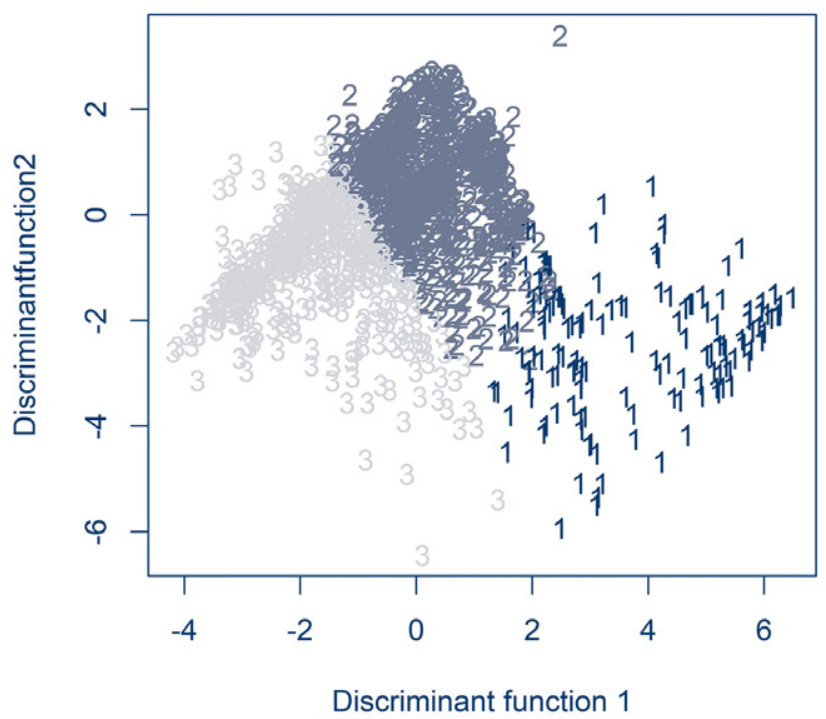


Tabla 9. Orden de los procesos didácticos del cluster 1 (n=1222)

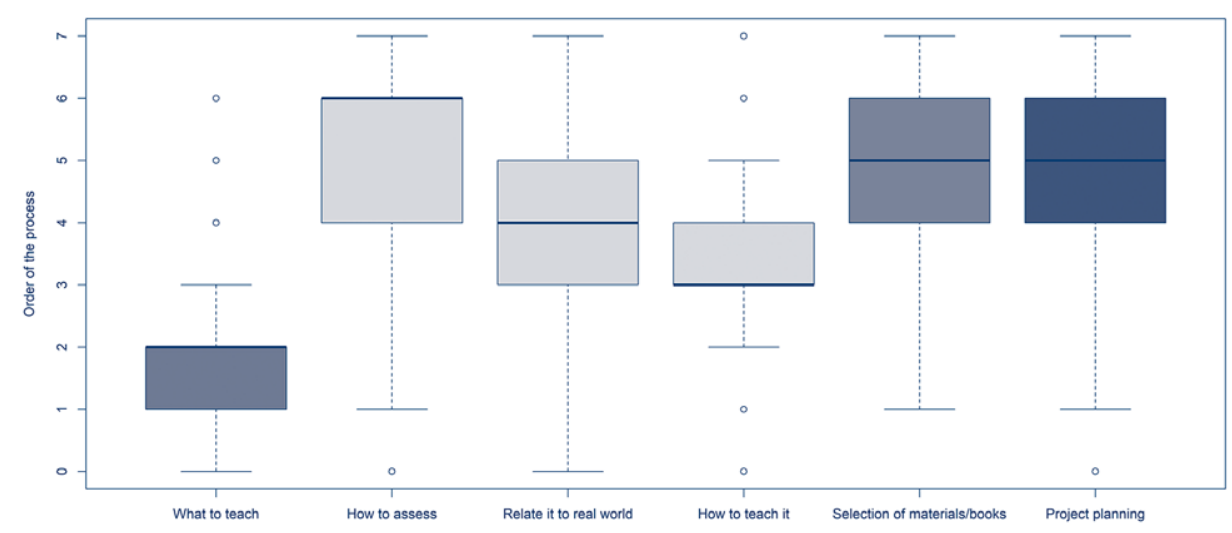

Tabla 10. Orden de los procesos didácticos del cluster 2 (n=188)

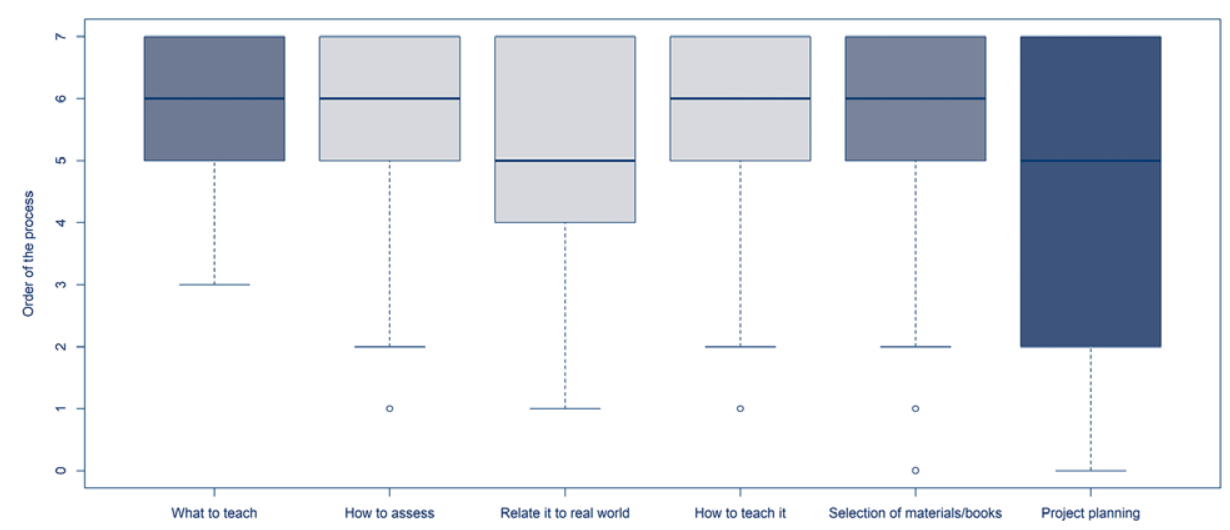

Tabla 11. Orden de los procesos didácticos del clúster 3 (n=606)

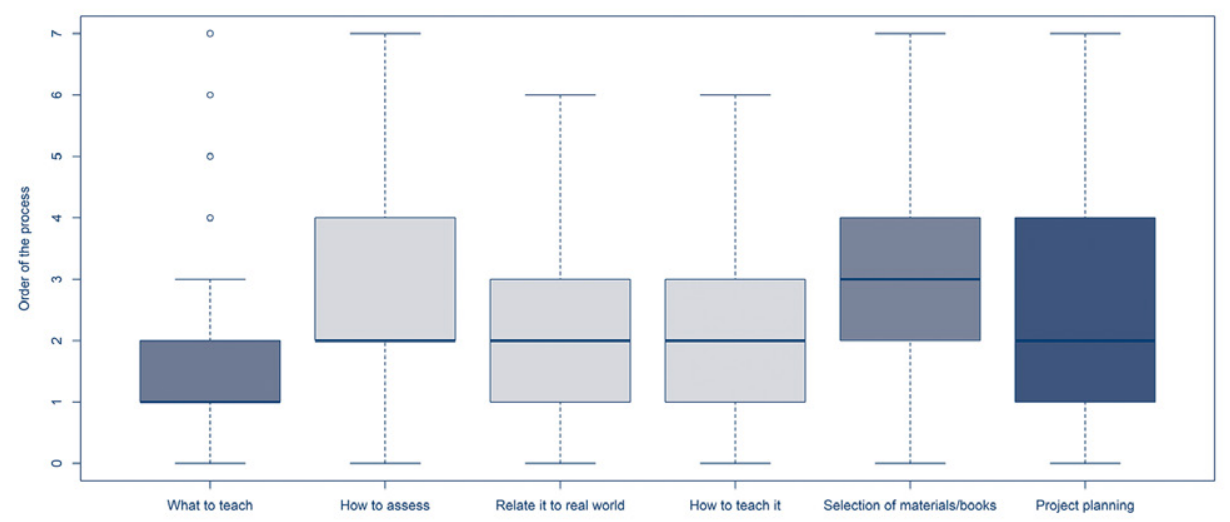


A partir del análisis de k-medias es posible afirmar la existencia de tres principales estilos de planeación (basados en el ordenamiento y priorización cognitiva de los diferentes procesos didácticos). Los docentes del cluster 1 -que parecen ser la gran mayoría, $\mathrm{n}=1222-$ $(60.62 \%)$ se relacionan más con una planeación de tipo forward design, donde el segundo proceso a definir son las actividades para enseñar el tema, acabando con la definición de «cómo evaluar». Esto es consistente con la racionalidad tyleriana explicada en la revisión de la literatura. En contraste, los docentes del cluster $3(n=606,30.06 \%)$ parecen referirse a una mezcla entre forward, central y backward. Es posible notar que los docentes del cluster 3 parecen privilegiar la definición de «cómo evaluar» como el segundo proceso didáctico (alineado con la planeación backward design). En contraste, el cluster $2(\mathrm{n}=188,9.33 \%)$ se representa por docentes que confunden o alteran la interpretación, ya que no parecen ser capaces de: ya sea (a) entender la instrucción proporcionada en el cuestionario; (b) poseen un estilo de planeación no contemplado por la forma del análisis, o (c) no tienen claridad al planear a partir del ordenamiento de sus procesos didácticos.

Para este fin, los docentes del cluster 1 serán llamados «planeadores clásicos basados en objetivos», los docentes del cluster 2 se denominarán "planeadores sin claridad» y los docentes del cluster 3 se nombrarán como «planeadores mixtos basados en estándares».

\section{DISCUSIÓN}

El presente trabajo buscó caracterizar los estilos de planeación didáctica de docentes de una red de colegios particulares. Dicho esto, la caracterización se llevó a cabo a través de una aproximación cognitiva al estudio de la estilística de la planeación didáctica. Ello se refiere a estudiar la toma de decisiones realizada por los docentes en torno a los elementos didácticos que constituyen un plan didáctico.

Al comparar los hallazgos con los modelos prescriptivos de planeación revisados, es posible afirmar que los docentes llamados «planeadores clásicos basados en objetivos» son altamente congruentes con lo prescrito en la racionalidad tyleriana. En otras palabras, estos docentes ( $n=1222 ; 59.52 \%$ de la muestra) se caracterizan por definir primero los contenidos y objetivos educativos, para después decidir qué actividades de enseñanza realizarán, y por último, determinar qué métodos de evaluación emplearán.

Es interesante este fenómeno, ya que habla de la relevancia de Tyler 70 años después de la publicación original de su propuesta de planeación. Retomando a John (2006), se puede argumentar que, en efecto, su permanencia radica en su «elegante simplicidad». La lógica detrás de la racionalidad tyleriana confirma que, en efecto, los docentes estudiados prefieren especificar sus metas para saber si las alcanzan, que analizar su proceso de enseñanza-aprendizaje que prefieren dividirlo en sus componentes, y que también eligen pasar por el proceso racional de formular objetivos, decidir estrategias, seleccionar recursos y evaluar sus resultados para planear su clase.

Otro fenómeno interesante es el hecho que se debió eliminar del análisis la variable «diagnóstico de necesidades». A partir de los datos obtenidos es que puede evidenciarse que realizar un diagnóstico de necesidades no se correlaciona con cualquier otra toma de decisión sobre la planeación de la enseñanza. Aunque teóricamente se entiende -porque Taba (1962) propondría un diagnóstico previo a planear cualquier clase y que además se promueve-, este trabajo de investigación evidencia la falta de seguimiento a su modelo prescriptivo. También pudiera ser que este es un elemento de deseabilidad de los docentes (todos lo eligen) o simplemente que no forma parte o es complejo de estudiar desde este abordaje cognitivo. 
Por otro lado, también es interesante la presencia correlacional del proceso «definir cuál es el proyecto que los alumnos van a desarrollar» según el cluster donde tiene mayor presencia. En el cluster 2, el proyecto posee la mayor relevancia entre los planeadores sin claridad. De lo poco que se infiere conclusivamente es que los datos parecen indicar que estos docentes, aunque no se guían por ningún otro elemento, la sustancia que organiza la enseñanza es el proyecto de su clase. En cambio, en el cluster 3, el proyecto de clase también parece acompañar las demás decisiones, otorgando coherencia a la conclusión del estilo de planeación que sustentan los docentes a este cluster. Debido a la naturaleza «mixta» del grupo, es posible inferir que algunos docentes tienden hacia la planeación por estándares (backward design), mientras que otros se orientan a una lógica Macdonaldiana (central design), y unos más a una tendencia basada en competencias -como Díaz-Barriga Arceo (2004) sostiene en su teoría sobre la planeación de la enseñanza situada, al optar por relacionar los objetivos educativos y contenidos de aprendizaje con la realidad social del educando-. En este sentido, el proyecto de la clase puede sostener las tres tendencias al explicarse que los docentes que planean por estándares suelen realizar proyectos como evidencia de los resultados de aprendizaje; los que siguen la lógica Macdonaldiana al utilizar el proyecto como actividad misma de enseñanza, y los que planean basados en competencias, al emplear el proyecto mismo como elemento de enseñanza activa y resolución de problemas de la realidad.

Ahora bien, un hallazgo interesante adicional es que, tras el análisis de componentes principales, se encontró una varianza explicada del $74 \%$. Esto abre amplias posibilidades ya que atestigua cuán prominente es la línea de investigación presentada. Ello abona a argumentar que la aproximación cognitiva a la estilística de la planeación didáctica es y puede ser investigada empleando los métodos aquí presentados.

Por último, cabe destacar que el presente trabajo de investigación tuvo ciertas dificultades teóricas y limitaciones operacionales. Por un lado, en la tónica de las dificultades teóricas, hubo una serie de obstáculos en la definición de las variables de planeación durante la construcción del instrumento de evaluación. Debido a la amplia variedad de modelos prescriptivos que existen $-y$ las sutiles diferencias de cada uno-, fue considerablemente complicado limitar conceptualmente el presente estudio. La primera dificultad consistió en que la literatura en español e inglés varía considerablemente. Como se mencionó durante la revisión de la literatura, la planeación didáctica es llamada de diferentes formas sin especificar, necesariamente, la variación entre sus connotaciones. Y la dificultad se incrementa a leer la literatura norteamericana, ya que utilizan términos que, en su traducción al español, implican significados distintos a los términos empleados originalmente para esos conceptos en nuestra lengua, y viceversa. Por ejemplo, el término "planeación didáctica» no existe en la literatura de habla inglesa, en cambio, debe buscarse como teacher planning o lesson planning. A partir de esta experiencia se recomienda que, para facilitar futuras investigaciones, se requiere primero realizar una homologación conceptual para evitar equivocaciones a la hora de la revisión.

Otra dificultad de la misma tónica consistió en la cercanía conceptual que tienen los estudios curriculares y los estudios didácticos en la temática de la planeación. Se concluyó que, debido a que en su mayoría la investigación relevante sobre planeación didáctica se ha hecho en la lengua inglesa, y que en dicha lengua no se emplea el término Didáctica como se emplea en la tradición latina, en muchas ocasiones la terminología se entrelaza, lo cual dificulta la diferenciación entre los campos de estudio. Sin duda, cada campo influye sobre el otro y las lógicas, en cuanto planeación, suelen ser muy parecidas. Es decir, tanto para la 
planeación curricular como para la planeación didáctica se utilizan los mismos elementos, pero en grados y contenidos distintos. Sin embargo, las decisiones suelen ser parecidas en cuanto a estructura y secuencia, pero no declarativamente. Por ello, aunque en este trabajo se mencionan los modelos prescriptivos con su empleo para planeación didáctica, en muchas ocasiones los autores plantean los modelos para la planeación curricular.

Adicionalmente a lo anterior, otra dificultad a lo largo del desarrollo del trabajo de investigación fue delimitar las variables detrás de una planeación didáctica. Aunque se enlistaron una serie de elementos reconocidos como generales y más importantes para toda evaluación, la realidad en el campo es que los docentes deben definir aún más elementos para que tenga sentido su clase. Todos estos elementos varían de clase a clase; sin embargo, es importante precisar que tanto la literatura, como las conversaciones con los docentes y especialistas, acordaron que relacionar el contenido con la vida real y definir un proyecto para la clase, son los elementos para concurrentes. Es por ello que se agregaron al instrumento.

Aunado a las dificultades teóricas, la limitación operacional consistió en que los docentes estudiados de educación básica y media superior laboran exclusivamente en colegios particulares. Y además, esos colegios particulares pertenecen a una misma red de colegios confesionales, lo cual tiende a modificar las pautas de acciones a un campo específico enmarcado en su cultura institucional. En cierto sentido, de esto podría concluirse que la muestra no es representativa a la docencia misma. Para futuras investigaciones se propondría realizar el mismo estudio contemplando una población más diversa para alcanzar mayores inferencias. Otra área de oportunidad es el estudio comparado de los procesos de planeación, dependiendo del nivel educativo.

En conclusión, debe reconocerse una vez más que el estudio presentado es de carácter exploratorio y descriptivo. Por lo tanto, esto indica que se trata de una primera aproximación hacia la caracterización de los estilos de planeación didáctica, basado en evidencia empírica, de cómo los docentes deciden planear. Sin duda, la metodología empleada es prometedora estadísticamente, sin embargo, existe amplio campo de oportunidad para experimentar con variables que evidencien, en mayor medida, los procesos de planeación didáctica. De igual manera es importante destacar el hallazgo de que el $60.61 \%$ de los profesores estudiados poseía una clara racionalidad clásica tyleriana o basada en objetivos; un $30.06 \%$ una mezcla entre estándares y objetivos, y sólo un $9.33 \%$ con una planeación que no es posible definir o que no se alinea a ninguno de los modelos teóricos descritos en este documento. 


\section{ANEXO}

\section{CUESTIONARIO PARA LA IDENTIFICACIÓN DE LOS ESTILOS DE PLANEACIÓN DIDÁCTICA}

\begin{tabular}{|c|c|c|c|}
\hline $\begin{array}{l}\text { Sección del } \\
\text { instrumento }\end{array}$ & Finalidad & Fraseo & Ítem \\
\hline \multirow[t]{7}{*}{$\begin{array}{l}\text { Estilos de } \\
\text { planeación. }\end{array}$} & \multirow{7}{*}{$\begin{array}{l}\text { Identificar } \\
\text { los estilos de } \\
\text { planeación de los } \\
\text { docentes. }\end{array}$} & \multirow{7}{*}{$\begin{array}{l}\text { Las siguientes actividades } \\
\text { son algunas que puedes } \\
\text { o no realizar cuando } \\
\text { planeas tus clases «desde } \\
\text { cero». Por favor ordénalas } \\
\text { según represente lo } \\
\text { que haces cuando } \\
\text { estás planeando. Si no } \\
\text { haces cierta actividad, } \\
\text { selecciona la opción } \\
\text { «no lo hago». Si haces } \\
\text { dos o más actividades al } \\
\text { mismo tiempo, puedes } \\
\text { seleccionar las opciones } \\
\text { en la misma columna. }\end{array}$} & $\begin{array}{l}\text { Seleccionar y evaluar } \\
\text { los materiales que } \\
\text { usaré. }\end{array}$ \\
\hline & & & $\begin{array}{l}\text { Definir cuál es el } \\
\text { proyecto que los } \\
\text { alumnos van a } \\
\text { desarrollar. }\end{array}$ \\
\hline & & & $\begin{array}{l}\text { Definir cómo voy a } \\
\text { enseñar la materia. }\end{array}$ \\
\hline & & & $\begin{array}{l}\text { Hacer un diagnóstico de } \\
\text { mis alumnos. }\end{array}$ \\
\hline & & & $\begin{array}{l}\text { Definir cómo lo voy a } \\
\text { evaluar. }\end{array}$ \\
\hline & & & $\begin{array}{l}\text { Definir cómo la materia } \\
\text { se relaciona con la vida } \\
\text { real. }\end{array}$ \\
\hline & & & $\begin{array}{l}\text { Definir qué voy a } \\
\text { enseñar. }\end{array}$ \\
\hline
\end{tabular}

\section{REFERENCIAS}

Ashcraft, N. (2014). Lesson planning. Estados Unidos: TESOL Publications.

Bobbit, F. (1918). The Curriculum. Boston: Houghton Miffin.

Bloom, B., et al (1956). Taxonomy of educational objectives: Handbook I, The cognitive domain. New York: David McHay \& Co.

Chevallard, Y. (1989). On didactic transposition theory: Some introductory notes. International symposium on selected domains of research and development in mathematics education, 51-62.

Clark, C., Yinger, R. (1979). Three Studies of Teacher Planning. Institute for Research on Teaching. (55), 1-26. 
Díaz-Barriga Arceo, F. (2004). Enseñanza situada: vínculo entre la escuela y la vida. México: McGraw Hill.

Eisner, E. (1967). Educational Objectives: Help or Hindrance? School Review, 75, 250-266.

Everitt, B. y Hothorn, T. (2010). A Handbook of Statistical Analysis Using R. New York: Taylor and Francis Group.

John, P. (2006). Lesson Planning and the student teacher: re-thinking the dominant model. Journal of Curriculum Studies, 38(4), 483-498.

Koeller, S., Thompson, E. (1980). Another Look at Lesson Planning. Educational Leadership. 37(8), 673-675.

Kyung Ko, E. (2012). What is your Objetive? Preservice Teacher's Views and Practice of Instructional Planning. The International Journal of Learning, 18(7), 89-100.

Macdonald, J. (1965). Myths About Instruction. Educational Leadership, 22(8), 571-576.

Macdonald, J., Wolfson, B., Zaret, E. (1973). Reschooling Society: A Conceptual model. Association for Supervision and Curriculum Development.

Medina Gual, L. (2011). Objetivos, Competencias y Estándares: Tres paradigmas pedagógicos para el uso de objetos de aprendizaje. Memorias del Segundo Coloquio de Ambientes Virtuales y Objetos de Aprendizaje. Experiencias y Reflexiones, 52-64.

Monroy, M. (2009). La Planeación Didáctica. En Monroy, M., Contreras, O., Desatnik, O., Ávila, J. (ed.) (2009). Psicología Educativa (pp. 453-487). México: UNAM-Facultad de Estudios Iztacala.

Ramírez, R. (2005). Aproximación al concepto de transposición didáctica. Folios. 1(21), 33-45.

Richards, J. (2013). Curriculum Approaches in Language Teaching: Forward, Central, and Backward Design. RELC, 44(1), 5-33.

Taba, H. (1962). Curriculum Development, Theory and Practice. New York: Harcourt, Brace and World, Inc.

Tyler, R. (1950). Basic Principles of Curriculum and Instruction. Chicago: University of Chicago Press.

Wiggins, G. y McTighe, J. (2005). Understanding by design. Estados Unidos: ASCD.

Zabalza, M.A. (2004). Guía para la planificación didáctica de la docencia universitaria en el marco del EEES. La Coruña: Universidad de la Coruña.

Zahorik, J. (1975). Teachers' Planning Models. Educational Leadership, 33(2), 134-139. 